\title{
Olyra bahiensis (Poaceae - Olyreae): uma nova espécie para a mata atlântica do estado da Bahia, Brasil
}

\author{
REYJANE PATRÍCIA DE OLIVEIRA ${ }^{1,3}$ e HILDA MARIA LONGHI-WAGNER ${ }^{2}$
}

(recebido: 28 de abril de 2005; aceito: 8 de dezembro de 2005)

\begin{abstract}
Olyra bahiensis (Poaceae - Olyreae): a new species from the Atlantic rain forest of the State of Bahia, Brazil). A new species of Olyra is described and illustrated. Olyra bahiensis R.P.Oliveira \& Longhi-Wagner is related to O. ciliatifolia Raddi (widely distributed in South America), and to O. juruana Mez, O. amapana Soderstr. \& Zuloaga, and O. loretensis Mez (occurring in the Amazonian Basin), sharing the presence of trichomes covering completely the female floret. The new species occurs in the Atlantic rain forest of Southern Bahia, in the same area where other many species of grasses are found. The populations of $O$. bahiensis present small number of individuals, growing in shaded areas associated with cocoa plantations. An analytical key for $O$. bahiensis and allied species is also presented.
\end{abstract}

Key words - Atlantic rain forest, Brazil, Olyra, Poaceae, taxonomy

RESUMO - (Olyra bahiensis (Poaceae - Olyreae): uma nova espécie para a mata atlântica do estado da Bahia, Brasil). Uma nova espécie de Olyra é descrita e ilustrada. Olyra bahiensis R.P.Oliveira \& Longhi-Wagner é relacionada à $O$. ciliatifolia Raddi (amplamente distribuída na América do Sul) e à O. juruana Mez, O. amapana Soderstr. \& Zuloaga e O. loretensis Mez (ocorrentes na região Amazônica), pela presença de tricomas recobrindo totalmente o antécio feminino. A nova espécie ocorre na mata atlântica do sul da Bahia, na mesma área onde também são encontradas várias espécies endêmicas de gramíneas. As populações de $O$. bahiensis apresentam pequeno número de indivíduos, os quais habitam áreas sombreadas em remanescentes de matas úmidas associadas ao cultivo de cacau. Uma chave analítica para $O$. bahiensis e espécies relacionadas é também apresentada.

Palavras-chave - Brasil, mata atlântica, Olyra, Poaceae, taxonomia

\section{Introdução}

A tribo Olyreae inclui os bambus herbáceos, popularmente conhecidos como "taquaras" os quais são importantes componentes do estrato herbáceo das florestas neotropicais. A mata atlântica representa o principal centro de diversidade do grupo (Soderstrom et al. 1988, Clark 1990), especialmente a região compreendida entre o sul da Bahia e o norte do Espírito Santo.

Olyra L. é o gênero que apresenta distribuição mais ampla dentro da tribo, além do maior número de espécies da subtribo Olyrinae, cerca de 23 (Judziewicz \& Zuloaga 1992). É também um dos gêneros mais heterogêneos e que apresenta maior variabilidade morfológica, sendo, provavelmente, polifilético (Judziewicz et al. 1999). Muitas espécies descritas como parte de Olyra têm

\footnotetext{
1. Programa de Pós-Graduação em Botânica, Universidade Estadual de Feira de Santana, Departamento de Ciências Biológicas, Rodovia BR 116N km 3, 44031-460 Feira de Santana, BA.

2. Universidade Federal do Rio Grande do Sul, Departamento de Botânica, Av. Bento Gonçalves 9500, 91501-970 Porto Alegre, RS.

3. Autor para correspondência: rpolliveira@yahoo.com
}

sido transferidas para outros gêneros da tribo Olyreae, como Agnesia lancifolia (Mez) Zuloaga \& Judz. (Zuloaga \& Judziewicz 1993), Cryptochloa capillata (Trin.) Soderstr. (Soderstrom 1982), e quatro espécies de Parodiolyra Soderstrom \& Zuloaga (Soderstrom \& Zuloaga 1989, Zuloaga \& Davidse 1999).

Ao contrário da maioria dos gêneros de Olyreae, que apresenta pouca variação nos caracteres reprodutivos, Olyra tem sua taxonomia baseada principalmente na morfologia, textura e indumento do antécio feminino. Embora ainda não existam estudos sobre a filogenia do gênero, Judziewicz \& Zuloaga (1992) reuniram grupos de espécies com determinados caracteres morfológicos em comum, que provavelmente correspondem a grupos naturais, segundo os mesmos autores. Entretanto, não agruparam as espécies de Olyra que apresentam o antécio feminino totalmente recoberto por tricomas: $O$. ciliatifolia Raddi, O. juruana Mez, O. amapana Soderstr. \& Zuloaga e O. loretensis Mez, além da nova espécie aqui descrita.

\section{Resultados e Discussão}

Olyra bahiensis R.P. Oliveira \& Longhi-Wagner, sp. nov. Tipo: BRASIL: BAHIA: Una, km 15.6 da rodovia 
São José/Una, 10 km SE até São José, 21-II-1986, T.S. dos Santos \& E.J. Judziewicz 4113 (holótipo CEPEC, isótipo US, n.v.).

Figura 1

Olyrae juruanae Mez et $\mathrm{O}$. amapanae Soderstrom \& Zuloaga anthoecio femineo omnino piloso affinis, sed glumis spiculae femineae inaequalibus, infera aristata differt. Olyrae ciliatifoliae Raddi anthoecio piloso et gluma infera aristata etiam affinis, sed panicula contracta, spiculis femineis pilosis et trichomatibus anthoecii feminei longioribus et densioribus differt. Anthoecio femineo piloso etiam Olyrae loretensis Mez affinis sed panicula contracta et culmis monomorphis differt.

Plantas perenes, cespitosas, 27-50(-100) cm compr., rizomas pouco desenvolvidos, colmos monomórficos, 1-3 mm diâm., não ramificados, esparsa até densamente pilosos, 6-11 folhas; nós pubescentes, escuros; cicatriz infra-nodal ausente. Bainhas foliares densamente pilosas nas margens e no ápice, menos freqüentemente em toda a extensão; lâminas $6,5-12(-15) \times 1,5-2,3(-4,7) \mathrm{cm}$, lanceoladas ou oval-lanceoladas, base fortemente assimétrica, com um lado arredondado e outro obtuso, ápice agudo, às vezes acuminado, face adaxial escabra na região da nervura central ou glabra, face abaxial hirsuta, margens escabras; pseudopecíolo 1-4 mm compr., densamente piloso; lígula membranoso-ciliada, 0,3-0,5 mm compr. Panícula 6-8 × 1-2(-3) cm, contraída, terminal, 1-4 por colmo; ramos inferiores alternos, apenas com espiguetas masculinas; ramos superiores com 1 a várias espiguetas femininas; ráquis achatada, pubescente. Espiguetas femininas 13-18(-21) $\times$ 2,5-2,8 mm, ovais ou oval-lanceoladas, pubescentes ou, menos freqüentemente, apenas escabras; pedicelo piloso ou escabro; glumas 6,5-18(-21) mm compr., membranosas, pubescentes ou escabras, (5)7-9nervadas, a inferior mais longa que a superior, aristada, arista 7-14 mm compr., a superior geralmente acuminada, às vezes aristada, arista com $2-3 \mathrm{~mm}$ compr.; antécio 5,8-6,5 × 1,6-1,8 mm, sem estípete na base, ovallanceolado, esbranquiçado, não se tornando maculado na maturação, piloso em toda a extensão, tricomas longos, densos; cariopse não vista. Espiguetas masculinas 4-5,7 $\times$ 0,7-1 mm, lanceoladas, amareladas; pedicelo piloso; glumas ausentes; lema acuminado ou curtamente aristado, arista até $1 \mathrm{~mm}$ compr., pubescente ou raramente escabro, 3-5(-7)-nervado. Estames 3.

Material adicional examinado: BRASIL. BAHIA: Buerarema, rodovia Buerarema - São José da Vitória, (BR 101), ramal à esquerda, ca. 15 km de Buerarema, entrada à direita ca. 9,5 km, Fazenda Santa Rosa, 5-II-2003, P. Fiaschi et al. 1271 (CEPEC, NY, SPF). Ilhéus, área do CEPLAC, Quadra D (cultivada), 13-III-2001, R.P. Oliveira \& H.M. Longhi-Wagner 712 (CEPEC, HUEFS). Una, 21-IV-1976, C.E. Calderón et al. 2382 (B, CEPEC, K, SP); km 15,6 da rodovia São José/Una, 21-II-1986, T.S. dos Santos \& E.J. Judziewicz 4032 (CEPEC), 4267 (CEPEC, HUEFS, K, MBM); ca. $9 \mathrm{~km}$ da BR 101, na estrada São José da Vitória para Una, 31-X-2003, R.P. Oliveira et al. 977 (CEPEC, RB, SP); ca. 5 km SE, estrada para São José do Macuco, 14-V-1991, A.M. Carvalho et al. 3336 (CEPEC); Serra Javi, 28-II-1986, T.S. dos Santos et al. 4071 (CEPEC).

Olyra bahiensis é morfologicamente relacionada com o grupo de espécies de Olyra que apresenta antécio feminino totalmente recoberto por tricomas. Diferencia-se das mesmas pela morfologia da inflorescência e das espiguetas femininas e masculinas. A inflorescência contraída e sem ramificações de O. bahiensis (figura 1), com espiguetas femininas agrupadas na metade apical da panícula e as masculinas na metade basal, é um dos caracteres que mais a aproxima de $O$. juruana. Esse padrão tem sido encontrado em espécies de Parodiolyra, nas quais, entretanto, o pedicelo das espiguetas femininas apresenta-se filiforme, enquanto que em Olyra, ele é clavado (Oliveira \& Longhi-Wagner 2001a, b). Em Olyra, o padrão de distribuição das espiguetas é variado, sendo mais freqüente aquele encontrado em $O$. ciliatifolia e $O$. loretensis (panícula muito ramificada, com uma espigueta feminina terminal em cada ramo, acompanhada de várias espiguetas masculinas adjacentes).

Pelos caracteres das espiguetas femininas, com glumas desiguais, a inferior maior que a superior e longamente aristada, Olyra bahiensis assemelha-se a O. ciliatifolia, sendo as glumas acuminadas e subiguais nas demais espécies. As duas diferem, entretanto, pelo indumento das espiguetas femininas, das glumas e do antécio, apresentando tricomas mais longos e densos em $O$. bahiensis. Quanto às espiguetas masculinas, são menores, pubescentes e sem arista no lema (ou quando presente, a arista é menor que $1 \mathrm{~mm}$ de comprimento) na nova espécie, enquanto que em $O$. ciliatifolia os lemas apresentam aristas de 1-3 $\mathrm{mm}$ de comprimento A coloração das espiguetas masculinas é totalmente amarelada em $O$. bahiensis e tipicamente avermelhada ou vinácea em $O$. ciliatifolia.

Vegetativamente, os indivíduos de Olyra bahiensis podem ser reconhecidos pelas bainhas foliares 


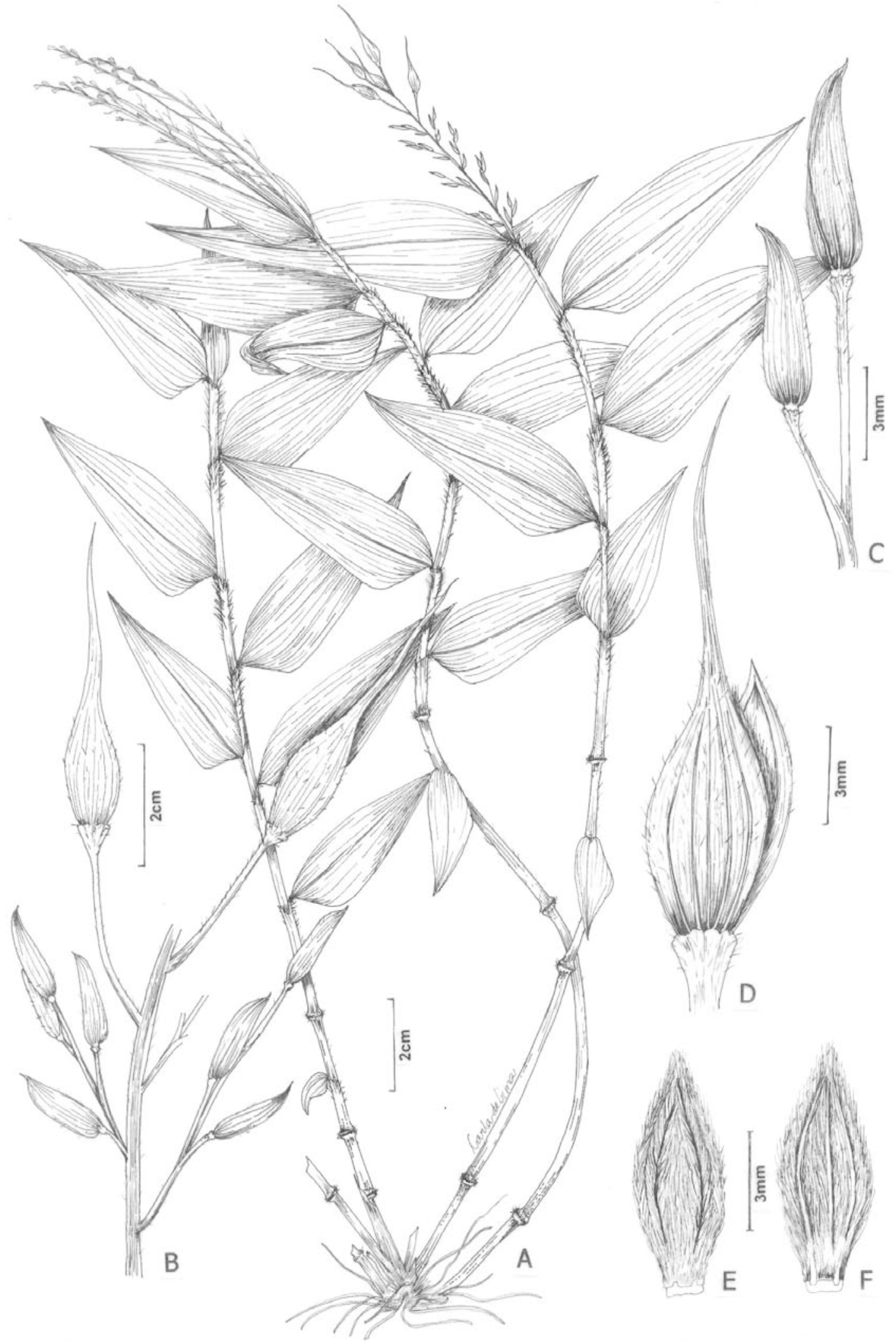

Figura 1. Olyra bahiensis. A. Hábito. B. Detalhe da inflorescência. C. Espiguetas masculinas. D. Espigueta feminina. E. Antécio feminino em vista ventral. F. Antécio feminino em vista dorsal (T.S. dos Santos \& E.J. Judziewicz 4113).

Figure 1. Olyra bahiensis. A. Habit. B. Inflorescence. C. Male spikelets. D. Female spikelets. E. Female anthecium in ventral view. F. Female anthecium in dorsal view (T.S. dos Santos \& E.J. Judziewicz 4113). 
densamente pilosas até vilosas e lâminas hirsutas na face abaxial, sendo glabras ou escabras nas espécies relacionadas. Na natureza, as lâminas foliares desta nova espécie apresentam uma conspícua coloração verde-escura brilhante na face adaxial, enquanto a face abaxial é glauca.

Chave para as espécies de Olyra relacionadas a O. bahiensis

1. Colmos dimórficos; inflorescências nascendo em colmos diferenciados, basais, com folhas reduzidas O. loretensis (Amazônia)

1. Colmos monomórficos; inflorescências nascendo em colmos não diferenciados, folhas semelhantes nos colmos vegetativos e nos reprodutivos

2. Inflorescência laxa, com uma espigueta feminina terminal em cada ramo da panícula e as masculinas adjacentes; lemas das espiguetas masculinas com aristas de 2-3 mm compr. O. ciliatifolia (América do Sul)

2. Inflorescência contraída, as espiguetas femininas agrupadas na metade apical da panícula e as masculinas na metade basal; lemas das espiguetas masculinas acuminados ou com aristas de até $1 \mathrm{~mm}$ compr.

3. Glumas das espiguetas femininas desiguais, a inferior mais longa que a superior, com arista de 7-4 mm compr. O. bahiensis (Bahia)

3. Glumas das espiguetas femininas subiguais, acuminadas a subuladas, não aristadas.

4. Inflorescência 1(-4) por colmo . O. juruana (Amazônia Peruana e oeste do Brasil) 4. Inflorescências cerca de 10 por colmo O. amapana (Amapá)

Olyra bahiensis é endêmica dos municípios de Una e Buerarema, área de Mata Atlântica de encosta no sul da Bahia. Essa área tem se destacado pela grande diversidade de espécies, principalmente de grupos bambusóides herbáceos, entre elas Pariana carvalhoi R.P.Oliveira \& Longhi-Wagner, recentemente descrita (Oliveira et al. 2004) e Anomochloa marantoidea Brongn. (Anomochlooideae), que emerge como basal na família em todas as análises filogenéticas que têm sido publicadas (GPWG 2001). Essas espécies apresentam endemismo restrito e suas populações incluem poucos indivíduos, os quais habitam áreas sombreadas em remanescentes de matas úmidas associadas ao cultivo de cacau. Além disto, ocorrem fora de unidades de conservação, submetidas ao crescente desmatamento que vem ocorrendo na Mata Atlântica sul-baiana. Devido a isso, podem ser consideradas criticamente ameaçadas, conforme os critérios estabelecidos pela IUCN (1994).

O material R.P. Oliveira \& H.M. Longhi-Wagner 712 (HUEFS) representa um indivíduo cultivado em uma área de mata higrófila do Centro de Pesquisas do Cacau, Ilhéus - BA, área onde também Anomochloa marantoidea se encontra em cultivo.

Agradecimentos - À Universidade Estadual de Feira de Santana pelo apoio financeiro. Ao Herbário do Centro de Pesquisas do Cacau (CEPEC/CEPLAC), especialmente ao Dr.
André M.V. de Carvalho (in memoriam) e Jomar G. Jardim pelo auxílio nas coletas. Ao Conselho Nacional de Desenvolvimento Científico e Tecnológico (CNPq) pelas bolsas concedidas (de Doutorado à primeira autora e Produtividade em Pesquisa à segunda autora).

\section{Referências bibliográficas}

CLARK, L.G. 1990. Diversity and biogeography of neotropical bamboos (Poaceae: Bambusoideae). Acta Botanica Brasilica 4:125-132.

GPWG (The Grass Phylogeny Working Group). 2001. Phylogeny and subfamilial classification of the grasses (Poaceae). Annals of the Missouri Botanical Garden 88:373-457.

IUCN. 1994. The world list of threatned trees. World Conservation Union Press. Appendix 4:629-643.

JUDZIEWICZ, E.J.L., CLARK, L.G., LONDOÑO, X. \& STERN, M.J. 1999. American bamboos. Smithsonian Institution Press, Washington.

JUDZIEWICZ, E.J.L. \& ZULOAGA, F.O. 1992. Olyra davidseana (Poaceae: Bambusoideae: Olyreae), a new species from Brazil. Systematic Botany 17:25-28.

OLIVEIRA, R.P. \& LONGHI-WAGNER, H.M. 2001a. Olyra L. In Flora Fanerogâmica do Estado de São Paulo (M.G.L. Wanderley, G.J. Shepherd \& A.M. Giulietti, orgs.). v. 1: Poaceae. Hucitec, São Paulo, p.45-47.

OLIVEIRA, R.P. \& LONGHI-WAGNER, H.M. 2001 b. Parodiolyra Soderstr. \& Zuloaga In Flora Fanerogâmica do Estado de São Paulo (M.G.L. Wanderley, G.J. Shepherd \& A.M. Giulietti, orgs.). v. 1: Poaceae. Hucitec, São Paulo, p.47-48. 
OLIVEIRA, R.P., LONGHI-WAGNER, H.M. \& HOLLOWELL, V.C. 2004. A new species of Pariana Aubl. (Poaceae: Bambusoideae: Olyreae) endemic to the Atlantic moist forest in the State of Bahia, Brazil. Novon 14:208-211.

SODERSTROM, T.R. 1982. New species of Cryptochloa and Piresia (Poaceae: Bambusoideae). Brittonia 34:199-209.

SODERSTROM, T.R. \& ZULOAGA, F.O. 1989. A revision of the genus Olyra and the new segregate genus Parodiolyra (Poaceae: Bambusoideae: Olyreae). Smithsonian Contributions to Botany 69:1-79.
SODERSTROM, T.R., JUDZIEWICZ, E.J.L. \& CLARK, L.G. 1988. Distribution patterns in Neotropical bamboos. In Proceedings of a Workshop on Neotropical Distribuition Patterns (W.R. Heyer \& P.E. Vanzolini, eds.). Academia Brasileira de Ciências, Rio de Janeiro, p.120-156.

ZULOAGA, F.O. \& DAVIDSE, G. 1999. Anew species and a new combination in the genus Parodiolyra (Poaceae: Bambusoideae: Olyreae). Novon 9:587-591.

ZULOAGA, F.O. \& JUDZIEWICZ, E.J.L. 1993. Agnesia, a new genus of Amazonian herbaceous bamboos (Poaceae: Bambusoideae: Olyreae). Novon 3:306-309. 